\title{
COMENTARIOS ACERCA DE \\ GUAZUMA ULMIFOLIA (STERCULIACEAE)
}

por CARMEN L. CRISTOBAL*

\section{Summary}

The typification of Theobroma Guazuma L. 三 Guazuma ulmifolia Lam. is discussed, and with the selection of a specimen from the Hortus Cliffortianus Herbarium, the synonymy of Guazuma tomentosa H.B.K. with G. ulmifolia, as established by Fawcett \& Rendle, Standley and Robyns, is confirmed. Within the species two varieties are recognized, separated mainly on the basis of fruit characteres, var. ulmifolia with the capsule remaining closed at maturity, and var. tomentella K. Schum. with the capsule incompletely dehiscent by five slits not wide enough to allow the seeds to escape; to a certain extent these differences are correlated with differences in shape of fruit, and outline and indument of the leaves. For both varieties the chromosome number $2 \mathrm{n}=16$, previously mentioned for G. ulmifolia, is established. A list of names proposed within the complex is appended, with comments on their probable identities, as appears from the information at present available.

Guazuma ulmifolia Lam. es una especie americana muy difundida, su área se extiende desde México hasta el NE de Argentina y

* Instituto de Botánica del Nordeste. Casilla de Correo 209, 3400 Corrientes, Rep. Argentina. Miembro de la Carrera del Investigador Científico y Tecnológico, CONICET.

Agradezco al Dr. TM. Pedersen la consulta de material en herbarios europeos y sus valiosas sugerencias. 
suele cultivarse en el viejo.mundo. L,os frutos cerrados o con cinco grietas, o sea incompletamente loculicidas, y la variabilidad de la forma, tamaño e indumento de las hojas se ha valorizado de diversas maneras como se resume a continuación.

linnaeus en su Species plantarum (1753, 782), ai publicar Theobroma (ruazuma cita en primer término su descripción anterior del Hortus cliffortianus $(1738,379)$, en la que se refiere a un árbol procedente de Jamaica, con hojas villosas y frutos verrucosos,..." “ $/ n$ ter lubercula cribi instar perforata... divisa en quinque loculamente interne... ". in sus dos okras cita una lámina de Plumier (1.703,36.t. 18). donde sc representa un fruto entero y otro en corte y ademas detalles florales que Linnaeus objeta ya que realmente pertenecen al género Corchorus. Fn las mismas obras Linnatus hace referencia también a Plukenet. Sloane y Ray. El primero de tllos (3691, t. 77, f. 2 y 1696, 92) describe igualmente una planta con fruto intero. dividido intenormente en cinco lóculos. I,a ilustración muestra una rama. un fruto completo sin aberturas y el mismo an corte lon gitudinal. Sloanc $(1696,135 ; 1725,18)$ y Ray $(1704.11)$ describen los frutos ron los mismos términos que Plukenct y a as hojas como lanuginosas.

Lamarck $(1789,52)$, al aceptar el nombre Guazuma propuesto por Plumier y validado por Miller (1754.596), (red el binomio Guazuma ulmefolea como nucvo nombre para Theobroma Ciuazuma L. Lamarek hace referencia a linnaeus, pero deseribe un rjemplar cultivado en il Jardín Real con fruto "...profondémenl geren" en dehors..." us decir agrietados.

lin llumboldt, Bonpland \& Kunth (1823 3320) se publica fiuazuma lomencosa H.R.K sobre la base de Iren ejemplares que se describer separadamente. Lon dos primeros, uno de Vompox (6o)

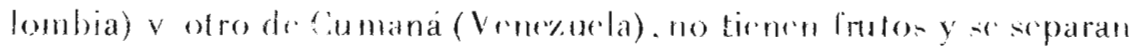
por diferencias on la forma y tamaño de las hojas fid tererero luen

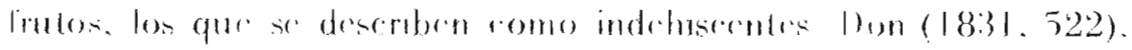

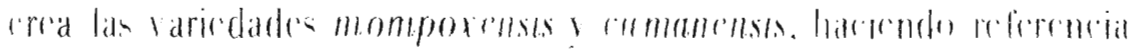
a lat dos primeras descriperomes. 
Schumann $(1886,81)$ distingue en G. ulmifolia cuatro variedades entre el material de Brasil, teniendo en cuenta la densidad del indumento foliar y si el fruto es indehiscente o no. Las dos primeras glabra y tomentella poseen frutos dehiscentes, la variedad tomentosa (= G. tomentosa H.B.K.) con frutos indehiscentes y velutina, como el nombre lo indica, es la de mayor densidad del indumento y sin datos de frutos. Al final agrega la variedad Trianae de Colombia, de la que tampoco vio frutos y describe como tomentosa.

Freytag (1951) al revisar el género, considera a G. ulmifolia con frutos loculicidas. Tipifica la especie con un ejemplar que atribuye al Herbario Lamarck (St. Thomas s/n). Posiblemente contó con la foto F $35346 \mathrm{P}-\mathrm{JU}$ ?. Esta foto es de un ejemplar de Baudin quien coleccionó en las Antillas en 1796-98 (Lanjouw \& Stafleu 1954-60), es decir con posterioridad a la publicación de Lamarck. Sin embargo en el Herbario Lamarck hay dos ejemplares, uno con una breve descripción datada 1784 y con fruto que coincide con la descripción publicada por Lamarck (Microficha IDC 93/10 y 93/12!).

Freytag acepta G. tomentosa diferenciándola de G. ulmifolia principalmente por el fruto indehiscente.

Otros autores como Fawcett \& Rendle (1926, 157), Standley (1923, 809) y Robyns $(1964,103)$, no aceptan $G$. tomentosa, y el último de los autores sinonimiza las variedades de G. ulmifolia propuestas por K. Schumann.

\section{Observaciones}

Estructura del fruto

He comparado poblaciones, posiblemente las más australes del género, del extremo $E$ de la provincia de Formosa con frutos completamente cerrados (Fig. 1 A), con otras del NE de la provin- 
cia de Corrientes con frutos con cinco grietas (Fig. 1 F). Observé que los dos tipos de frutos son indehiscentes, ya que en los que tienen grietas, éstas son tan angostas que no permiten que las semillas queden en libertad. Debido a que las prominencias varían en forma y tamaño, remojé tanto frutos cerrados como agrietados con el fin de remover la capa superficial que está constituida por parénquima con grandes cavidades mucilaginosas. Simplemente cepillando, queda al descubierto la segunda capa que es leñosa constituída por una red fibrovascular, a través de la cual hasta pueden verse las semillas cuando el fruto está seco. Esta red tiene proyecciones hacia la superficie que son el "esqueleto" de las prominencias.

En los frutos cerrados (Fig. $1 \mathrm{~B}-\mathrm{C}$ ), las prominencias son generalmente cónicas, agudas, es decir que los cordones fibrovasculares van disminuyendo hacia la punta. Por otro lado, muchas veces las prominencias no son de tamaño uniforme, las que están sobre la vena media de cada carpelo son más robustas y entre ellas hay otras más pequeñas.

En los frutos agrietados (Fig. 1 G), el "esqueleto" de las prominencias es bastante uniforme en tamaño y más o menos plano, es decir los cordones que se dirigen hacia la superficie son más o menos del mismo largo. Lo más curioso es que en estos frutos la vena media de cada carpelo se bifurca en la base del fruto, lo que permite que a la madurez se produzca la gricta. En los bordes de cada grieta se alinean las prominencias.

En algunos ejemplares de herbario se pueden observar frutos donde la capa superficial blanda se ha contraído alrededor del esqueleto leñoso (Fig. 1 A). lin estos casos es posible ver la red basal fibrovascular a modo de criba.

Las diferencias señaladas en los frutos no siempre se pueden observar en los ejemplares do herbario, ya que éstos pueden tener frutos incompletamente maduros. En algunos casos al remover la capa superficial, recién he podido observar la bifurcación de la vena media, es decir el comienzo de la gricta. Por otro lado, aunque el "esqueleto" de las prominencias de los frutos agrietados sea plano, 


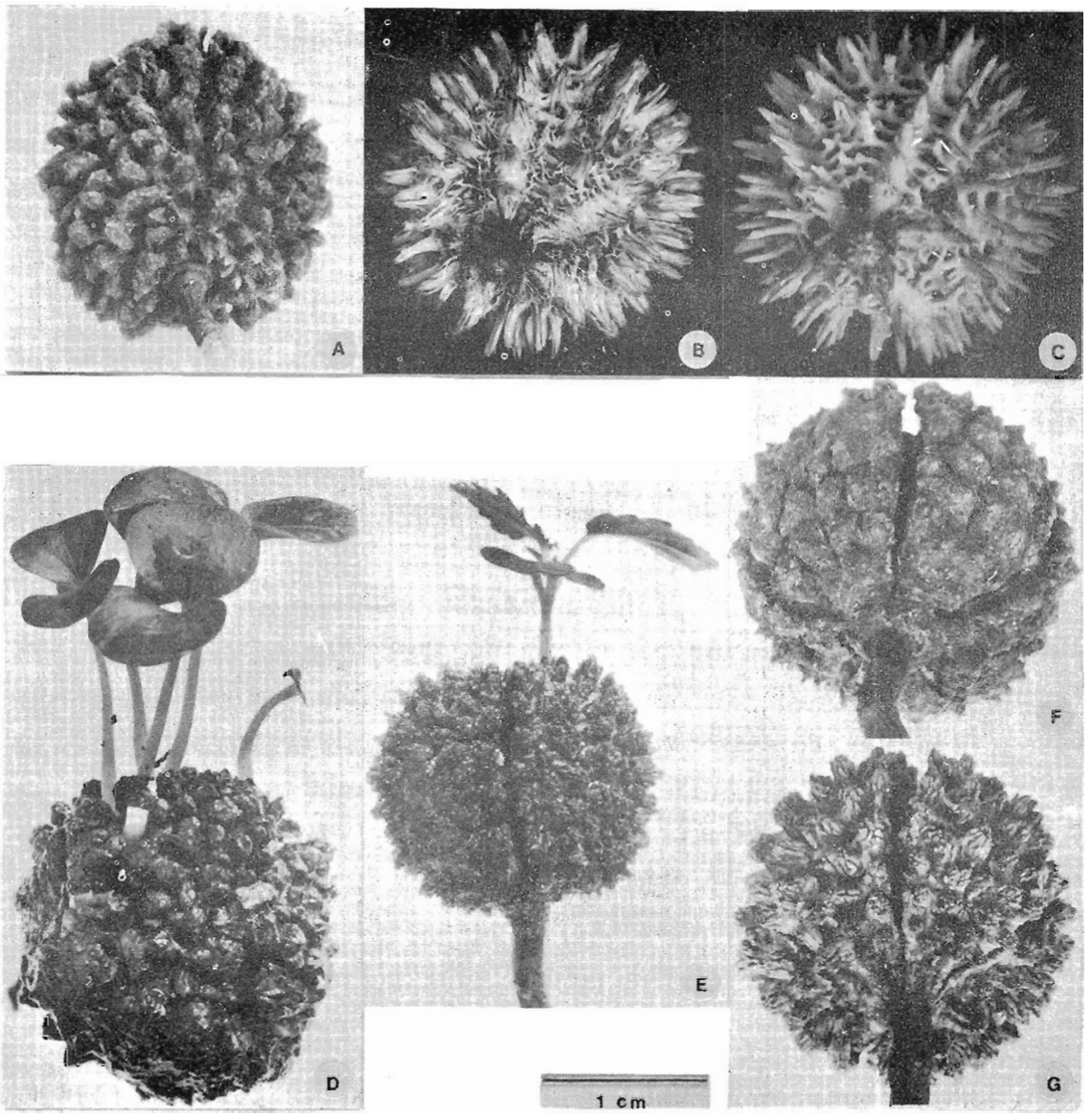

Fig. 1. A-D, Guazuma ulmifolia var. ulmifolia. A, fruto maduro completo. B-C, frutos sin la capa superficial. D, fruto durante la germinación de las semillas. (A, B, D: Cristóbal 2117, B: Stevens 3615). E-G, Guazuma ulmifolia var. tomentella. E, fruto du rante la germinación de las semillas. F, fruto maduro completo. G, fruto sin la capa superficial. (E: Krapovickas \& al. 41152, $\mathrm{F}-\overline{\mathrm{G}}$ : Cristóbal 20.58). 
en el fruto completo las prominencias terminan en una punta más - menos sobresaliente formada por tejido parenquimático (Fig. $1 \mathrm{~F})$. Finalmente, aunque en general en los frutos cerrados las prominencias sobre la vena media son más robustas, en ejemplares como de Paula 1828, las prominencias son agudas pero bastante uniformes en tamaño, es decir que las que están sobre la vena media no se destacan.

Cuando el fruto cae al suelo el pericarpo demora en degradarse. Las plántulas emergen a través de ia capa esclerosada (Fig. 1 D), o a través de las grietas (Fig. 1 E), es decir que ambos tipos de frutos son verdaderamente indehiscentes. Debajo de un árbol con frutos maduros, grupos de plántulas delatan la presencia de frutos enterrados.

Es posible que en la diseminación intervengan los animales. Los frutos son aceptados por el ganado, dato que ha sido registrado desde muy antiguo. Oviedo (1535) describe que los frutos remojados y machacados constituyen una mezcla que engorda fácilmente a los caballos. Las cápsulas molidas se usan como sustituto del maíz y el ganado busca los árboles para comer las cápsulas cuando falta el pasto (Sloane, 1725). Estos usos incluso han dado lugar al sinónimo genérico Bubroma Schreber.

Indumento foliar

El indumento foliar de C. ulmifolia varía en densidad, tamaño y tipos de pelos. Los pelos son generalmente estrellados, con diverso número de radios, y en cuanto al tamaño pueden ser diminutos, pequeños o medianos. En algunos ejemplares el tamaño es uniforme en toda la superficie foliar, en otros se encuentran pelos mucho más grandes dispersos entre los más pequeños. Excepcionalmente encontré predominancia de pelos simples en el epifilo, como por ejemplo en los ejemplares Schinini 4999 y 15151 de Paraguay.

El epifilo puede ser lustroso u opaco, pero hay grados interme- 
dios; puede ser glabro, con pelos sobre las venas principales o con pelos uniformemente dispersos en toda la superficie.

El hipofilo puede ser desde glabro hasta tomentoso, pasando por numerosos grados intermedios: pelos dispersos, o pelos entrecruzados pero que dejan ver la superficie de la epidermis. La densidad puede ser uniforme en toda la lámina o puede haber más pelos sobre las venas principales, o a los lados de las mismas o más raro en la axila de las ramificaciones.

He observado que la densidad varía también en distintos tipos de ramas de un mismo ejemplar, ambas con frutos (Cristóbal \& al. 2153).

Existe cierta correlación entre el indumento foliar y los tipos de frutos antes descriptos. En la gradación de densidad del indumento, las plantas con frutos cerrados llegan al grado máximo, y las de frutos agrietados llegan al grado mínimo. Sin embargo en los grados intermedios encontramos plantas con ambos tipos de frutos. Como ejemplo puedo citar ejemplares de Brasil como Irwin \& al. 15861 y 17101 , con frutos cerrados pero con epifilo lustroso, glabro y el hipofilo con escasos pelos dispersos. Como consecuencia, si contamos con un ejemplar de herbario con flores solamente, en muchos casos no es posible asegurar si se trata de una planta con frutos cerrados o agrietados.

\section{Cromosomas}

He contado $2 n=16$ cromosomas en plantas con ambos tipos de frutos ${ }^{1}$. Este número coincide con un recuento previo (Krishnappa \& al. 1980, 536), pero creo interesante ratificar el resultado en relación a las diferencias señaladas.

1 Agradezco a los integrantes del laboratorio de Citogenética del IBONE las facilidades brindadas. 
Los recuentos se hicieron en raicillas obtenidas al desenterrar frutos una vez que emergieron los cotiledones. El material fue pretratado con 8-hidroxiquinoleína, fijado en alcohol-acético 3:1, coloreado con Feulgen y aplastado en orceína acética. Los testigos son los siguientes: Cristóbal 2117 y 2058.

\section{Conchusiones}

En cuanto a la tipificación de G. ulmifolia, no se puede aceptar la elección de Freytag $(1951,220)$, de un ejemplar del Herbario Lamarck ?, s/n de St. Thomas, desde el momento que Lamarck da como sinónimo a Theobroma Guazuma L., es decir da un nuevo nombre a una especie lineana.

En el Herbario Lineano no existe un ejemplar previo a 1753 (Savage, 1945), por lo tanto para la tipificación se debe recurrir a los otros elementos de juicio citados por Linnaeus. Tanto en el Herbario Cliffortiano como en el Herbario Sloane hay ejemplares con frutos maduros (T.M. Pedersen, com. pers.). Por esta razón propongo como neotipo un ejemplar del Herbario Cliffortiano visto por Linnaeus y cuyos frutos coinciden con la descripción de este. autor, es decir enteros, sin grietas (l'ig. 2).

Por otro lado el material que vio Linnaeus es de Jamaica. Fawcett \& Rendle (loc. cit.), al describir e ilustrar detalladamente esta especie, mencionan frutos indehiscentes con numerosas perforaciones entre las prominencias, es decir que no vieron frutos agrietados entre el material de Jamaica.

Desde el punto de vista taxonómico considero que el tipo de fruto debe valorizarse, pero no en la medida aceptada por Freytag en su revisión del género, es decir como base para diferenciar dos especies. Me parece más adecuado cl criterio de Schumann (loc. cit., 81), que usa el nivel varietal. Sin embargo propongo aceptar solamente dos de las cinco variedades, que pueden diferenciarse fundamentalmente por el tipo de fruto y desvalorizo el indumento 


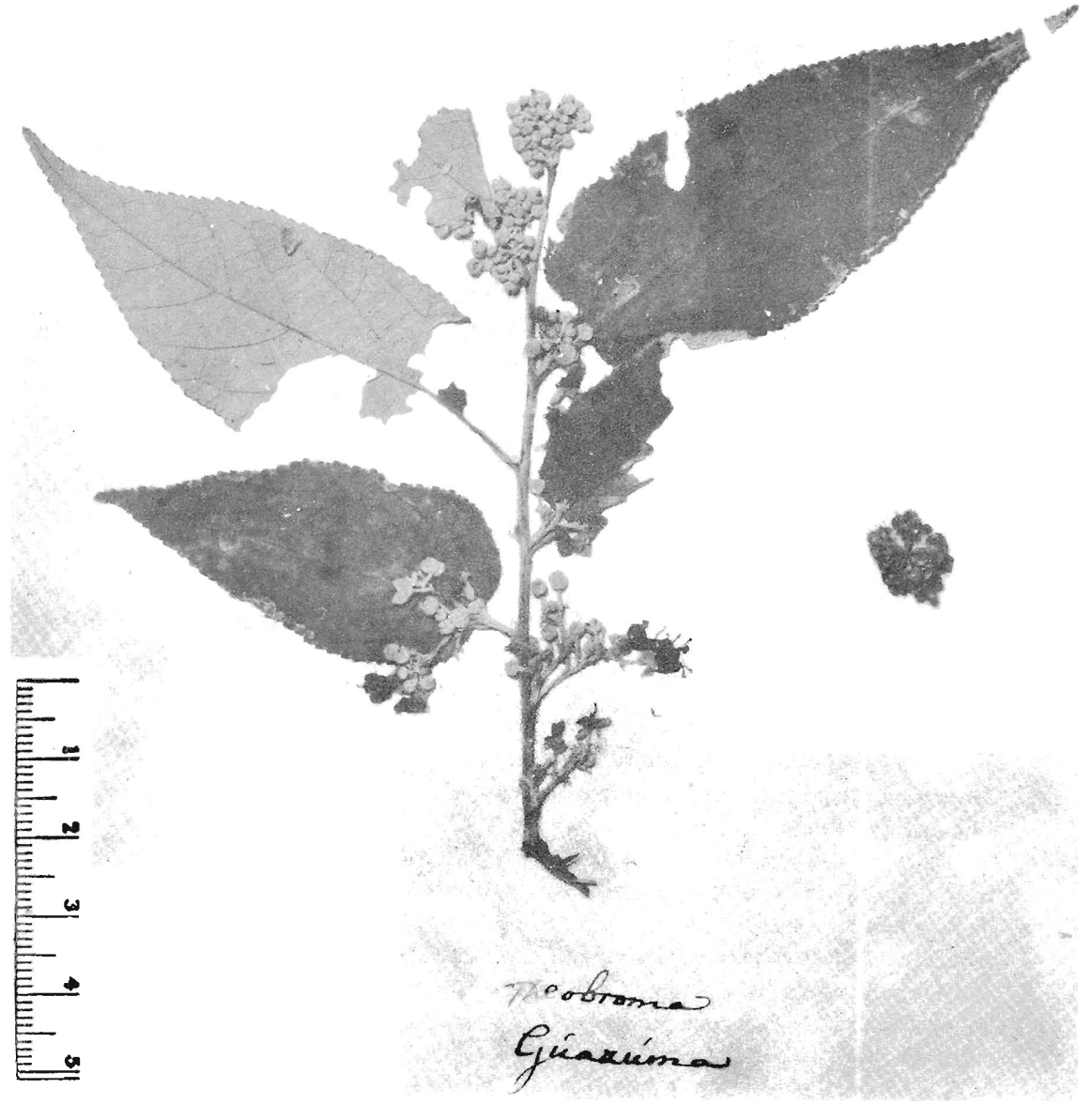

Fig. 2. Guazuma ulmifolia Lam.: neotipo de Theobroma Guazuma L., Clifford Herbarium, cortesía del British Museum (Natural History) London.

que este autor también usó para separar el resto de las variedades.

Las variedades aquí aceptadas pueden distinguirse de la siguiente manera: 
1. Frutos cerrados, o sea completamente indehiscentes, desde elipsoidales a esféricos. Epifilo por lo general no lustroso, a veces sublustroso raro lustroso; pelos estrellados diminutos o medianos, raro en su mayoría simples; dispersos o densamente dispuestos, raro sólo sobre las venas o glabro. Hipofilo con pelos estrellados diminutos o medianos, desde dispersos hasta cubriendo la superficie de la epidermis (tomentoso).

G. ulmifolia var. ulmifolia

1. Frutos con 5 grietas, o sea incompletamente loculicidas, desd e esféricos hasta complanados. Epifilo lustroso o sublustroso, con frecuencia glabro, o con pelos estrellados diminutos o medianos sobre las venas principales, o dispersos en toda la superficie y más abundantes sobre las venas. Hipofilo glabro, o con pelos estrellados diminutos o medianos sobre las venas principales y en la axila de las venas secundarias, o dispersos en toda la superficie.

G. ulmifolia var. tomentella

A continuación enumero cronológicamente los nombres involucrados con G. ulmifolia, y agrego mi interpretación de la sinonimia en los casos que he tenido su ficientes elementos de juicio.

1703 Guazuma arbor ulmi-folia, fructu ex purpura nigro. Plumier, Nov. pl. amer. 36 t. 18. "Guazuma est nomen Americanarum. Oviedo Hist. lib. 8 cap. 7" 三Guazuma ulmifolia var. ulmifolia.

1738. Theobroma foliis serratis L. Hort. cliff. 379. "Crescit in campestribus et sylvis apertioribus Jamaicae" $\equiv$ Guazuma ulmifolia var. ulmifolia.

1753. Theobroma Guazuma L. Sp. pl. 782. "Theobroma foliis serratis": "Habitat in Jamaicae campestribus" ĐGuazuma ulmifolia var. ulmifolia.

1789. Guazuma ulmifolia Lam. Encycl. 3.52, nuevo nombre para Theobroma Guazuma L. Sp. pl. 782.1753. Bubroma guazuma (L.) Willd. Sp. pl. 3(2): 1423.1803. Guazuma guazuma (L.) Cockerell, Bull. Torrey Bot. Club 19:95. 1892. Guazuma guazuma (L.) Cockerell $\alpha$ ulmifolia (Lam.) o.Kuntze, Revis. gen. pl. 3 (2): 24.1898.

1795-1796. Guazuma polybotria Cav. Icon. 3(2): 51, t, 299. "Habitat in Nova-Hispania" ".. pagina superiore nitida et scabriuscula, inferiore tomentosa..." No vió frutos. Bubroma polybotrium (Cav.) Willd. Enum. pl. 806.1809. = Guazuma ulmifolia var. ulmifolia.

1809. Bubroma Invira Willd Enum. pl. 806. 1809. "Habitat in Brasilia". "Dignositur facile glabritie foliorum, quae in juventute tantum leviter subpubescentia". Guazuma Invira (Willd.) G. Don Gen. hist 1:523. 1831. "Native of Brazil". Microficha Herb. Willd. $1027, \mathrm{n}^{\circ} 14349$ !. Rama sin frutos. = Guazuma ulmifolia var. tomentella K. Schum. 
1823. Guazuma tomentosa H.B.K. Nov. gen. sp. 5:320. "Crescit in ripa fluminis Magdalenae, prope Mompox alt. 40 hex.; item in Provincia Novae Andalusiae, prope Bordones". Bubroma tomentosum (H.B.K.) Spreng. Syst veg. 3: 332.1826. Guazuma tomentosa var. cumanensis G. Don, Gen. hist. 1: 523.1831. Guazuma tomentosa var. mompoxensis G. Don, Gen. hist. 1:523.1831. Guazuma ulmifolia var. tomentosa (H.B.K.) K. Schum., en Martius Fl. bras. 12(3): 81. 1886. Theobroma tomentosa (H.B.K.) Gómez, Anales Soc. Esp. Hist. Nat. 19: 217.1890. Guazuma Guazuma tomentosa (H.B.K.) O. Kuntze, Revis. gen. pl. 3(2): 24.1898. = Guazuma ulmifolia var. ulmifolia.

1826. Bubroma grandiflorum Willd. ex Spreng. Syst. veg. 3:332.1826. Theobroma grandiflorum (Willd. ex Spreng.) K. Schum. Fl. bras. 12(3): 76. 1886. Guazuma grandiflora (Willd. ex Spreng.) G. Don, Gen. hist. 1:523, 1831.

1827. Guazuma Bubroma Tussac, Fl. Antill. 4:69, t. 24.1827. No describe el indumento. En el dibujo del fruto en corte no se ven aberturas. En la descripción dice que el fruto interiormente se divide en 5 lóculos. Guazuma ulmifolia var. ulmifolia.

1831. Guazuma Blumii. G. Don, Gen. hist. 1: 523. "Native of Java". No describe frutos, por el indumento podría ser Guazuma ulmifolia var. ulmifolia.

184l. Guazuma parvifolia A. Richard, Hist. Phys. Cuba, Bot. Pl. vasc. 190. Describe el hipofilo tomentoso. = Guazuna ulmifolia var. ulmifolia.

1845. Guazuma utilis Poepp. \& Endl. Nov. gen. sp. pl. 3: 72-73. "Crescit in ripa fluminis Huallaga ad Yurimaguas". Tipo 2118 W!. Foto F 9638 B !. Ejemplar sin frutos; hojas con pelos estrellados diminutos, dispersos en ambas caras. = Guazuma ulmifolia var. tomentella?.

1852. Diuroglossum rufescens Turcz. Bull. Soc. Naturalistes Moscou 25 (2): 157. "Capsula ignota. Arbor Guayaquilensis. - foliis. - subtus dense stellato-tomentosis. Jameson coli. n. 399 et 51 9". = Guazuma ulmifolia var. ulmifolia.

1886. Cuazuma ulmifolia Lam. var. glabra. K. Schum., en Martius, Fl. bras. 12(3): 81. Freytag (1951, 220), establece como iectotipo a Poeppig 2118, ejemplar citado en primer término por $\mathrm{K}$. Schumann, sin embargo Freytag no le cita entre el material estudiado, y por otro lado Poeppig 2118 es el tipo de G. utilis, especie que K. Schumanr no vio e incluye en incertae sedis $=$ Guazuma ulmifolia var. tomentella.

1886. Guazuma ulmifolia Lam, var. tomentelia K. Scrum., en Martius, F1. bras. 12(3): 81. Freytag $(1951,220)$, establece como lectotipo: Peruvia orientalis ad Maynas: Poeppig sine numero, ejemplar citado por K. Schumann en primer término. No creo adecuada esta tipificación por tratarse de un ejemplar sin número posiblemente destruido en Berlín. Solicité a W ejemplares de Peoppig sin número de Maynas, pero no existen. Propongo por esta razón como lectotipo el ejemplar Glaziou 12455 (C!) citado por K. Schumann y con fru tos madu ros que concuerdan con la descripción.

1886. Guazuma ulmifolia Lam. var. Trianae K. Schum., en Martius, F1. bras. 12(3): 82. "Triana apud Antioquia". Foto I' 23831 !. Ejemplar sin frutos. Por la densidad del indumento = Guazuma ulmifolia var. ulmifolia.

1886. Guazuma ulmifolic Lam. var. velutina K. Schum., en Martius, F1. bras. 12(3): 81 . Lectotipo: "Brasiliae provinciis meridionalibus St. Hilaire". (Freytag, 1951, 220). K. Schumann no vio ejemplares con frutos. Según el indumento $=$ Guazuma ulmifolia var. ulmifolia. 
1907. Guazuma coriacea Rusby, Bull. New York Bot. Gard. 4:332. "(N²217)". Bolivia NY !. Frutos inmaduros, por la forma de las prominencias y por el indumento foliar $=$ Guazuma ulmifolia var. tomentella

\section{Material adicional seleccionado}

Guazuma ulmifolia var. ulmifolia: NICARAGLA. Managua, ca. $1.9 \mathrm{~km}$ W de Montelimar, ca. $11^{\circ} 49^{\prime} \mathrm{N}, 86^{\circ} 32^{\prime} \mathrm{W}, 31 . V$ III.1977. Stevens 3615 (MO, CTES). VENEZUELA. Zulia, Bolívar, en el Toro, sitio en la carretera El Consejo-Palito Blanco, en $\mathrm{km} 8$ al SSO de El Consejo, 22.II.1979, Bunting 7052 (CTES). BRASIL. Mato Grosso do Sul, Miranda, 8.IX 1985, de Paula 1828 (CTES). $30 \mathrm{~km} \mathrm{NE}$ of Brasilia, D.F., 14.V.1966, Irwin \& al. 15861 (CTES). Mato Grosso, ca. $25 \mathrm{~km} \mathrm{~S}$ of Xavantina, 13.VI.1966, Irwin \& al. 17101 (CTES). BOLIVIA. Beni, prov. Ballivian, Espíritu, en la zona de influencia del río Yacuma, 28.IX.1979, Beck 2510 (CTES). PARAGUAY. Asunción, Jandín Botánico, 22.VII.1972, Schinini 4999 (CTES, G, BAA). Chaco, Mayor Pedro Lagerenza, Parque Nac. Defensores del Chaco, Río Timane, 9.IV.1978. Schinini \& Bordas 15151 (CTES). ARGENTINA. Formosa, Paque Botánico Forestal Ing. Agrón. L. Tortorelli, ruta 11, Riacho Pilagá, 14.XI. 1986, Cristóbal \& al. 2117 (CTES, LIL, MO). Idem. 19.IV.1987, Cristóbal \& al. 2153 (CTES, MO).

Guazuma ulmifolia var. tomentella: PANAMA. Darien, Río Pirre, trail up river from house of Bartolo, 16.III.1973, Kennedy 2875 (MO). BRASIL, Pará, AMZA camp. 3-Alfa, $5^{\circ}$ 48'S, 50 33' W, 8.VI.1982, Sperling \& al. 5990 (CTES). Brasilia D.F., bacia do rio São Bartolomeu, 24.VI. 1980, Heringer \& al. 5132 (CTES). PARAGUAY. Depto. Central, Nemby, 15.VIII.1981, Vavrek 284 (CTES). ARGENTINA. Corrientes, Ituzaingó, ruta 38 y río Aguapey, 6.XII.1981, Tressens \& al. 1706 (CTES, C, MBM). Idem. 29.XI.1985, Cristóbal \& al. 2058 (CTES). Ea. Santa Rita, 56 4' W, $27^{\circ} 3^{\prime}$ S, 5.III.1987, Krapovickas \& al. 41152 (CTES, LIL, MO).

\section{Bibliografía}

Don, G. 1831. A general history of the dichlamydeous plants IXXVIII, 1-818.

Fawcett W. \& A.B. Rendle. 1926. Flora of Jamaica. Familia LXVI. Sterculiaceae. 5: 154-171.

Freytag, G.F. 1951. A revision of the genus (ruazuma. Ceiba 1(4): $193-225$. 
Glaziou, A.F.M. 1905. Plantae Brasiliae centralis a Glaziou lectae. Liste des plantes du Brèsil central recueillies en 1861-1895. Bull. Soc. Bot. France, Memoires 3: 52.

Humboldt, F.H. von, A. Bonpland \& C. Kunth. 1823. Nova genera et species plantarum. 5: (23) 320-322.

Krishnappa \& Munirajappa. 1980, en Löve, IOPB Chromosome number reports LXVIII. Tax on 29(4): 533-547.

Lamarck, J.B. de, 1789. Encyclopédie méthodique. Botanique.. $3: 52$.

Lanjouw, J. \& F.A. Stafleu. 1954. Index Herbariorum. Part II Collectors. Regnum Veg. 2: 1- 174. Utrecht.

Linnaeus, C. 1738. Hortus cliffortianus. 379.

\section{Species plantarum.782.}

Niller, P. 1754. The gardeners dictionary. Abridged. 2: 596. Ed. 4. London.

Oviedo y Valdés, G., Fernández de. 1535. Historia general y natural de las Indias, Islas y Tierra Firme del Mar Océano. Libr. 8, Cap. 7: "Del árbol llamado guazuma e de su fruto". En Biblioteca de Autores Españoles 117: 254. 1959. Ed. Atlas, Madrid.

Plukenet, L. 1691. Phytographia. t. 77, f.2. Londini. 1696. Almagestum. 92. Londini.

Plumier, Ch. 1703. Nova plantarum americanarum genera, ... 36, t. 18. Parisiis.

Ray: J. 1704. Historia plantarum 3:11.

Robyns, A. 1964. Sterculiaceae en Woodson, R.E. \& al. Flora of Panamá. Ann. Miss. Bot. Gard. 51(1-4): 69- 107.

Savage, S.A. 1945. A catalogue of the Linnaean Herbarium. 1-225. Schumann, K. 1886. Sterculiaceae, en Martius, C., Flora brasiliensis 12(3): 2-114, figs. 1--24.

Sloane, H. 1696. Catalogus plantarum quae in insula Jamaica sponte proveniunt. 135 .

1725. A Voyage to the island Madera, Barbados, Nieves, St. Christophers, and Jamaica. 2:18. 
Standley, P.C. 1923. Trees and shrubs of Mexico. Contr. U.S. Natl. Herb. 23(3): 517-848. 